\title{
Evaluation of Knee Kinematics in Single Radius versus Multi-Radii Total Knee Arthroplasty in India Population : A Randomized Control Trial
}

\author{
Lalit Maini $^{1}$, Ankur Sharma*1 ${ }^{*}$ Anshul Goel ${ }^{2}$, Amit Sharma $^{1}$, Surabhi Rohilla $^{1}$, Sunil Jha ${ }^{3}$, Ashish Indani ${ }^{4}$ and Srinivas \\ Reddy Boreddy ${ }^{4}$
}

${ }^{1}$ Department of Orthopaedics, LokNayak Hospital, India

${ }^{2}$ Department of Orthopedics, Wrightington Hospital, UK

${ }^{3}$ Biomedical Engineering, Indian Institute of Technology, India

${ }^{4}$ Medical Writing Domain, TATA Consultancy Services, India

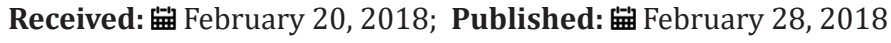

*Corresponding author: Ankur Sharma, LokNayak Hospital, orthopaedics, Bahadur Shah Zafar Marg, New Delhi, IN 110006, Tel: 01123232400; Email: lalit_maini@rediffmail.com

\begin{abstract}
Purpose: Single radius (SR) TKA implant designs are theoretically claimed to overcome "mid-flexion instability" and decrease quadriceps loading encountered in multiple radii (MR) designs. However, limited/no data is available to support SR over MR designs especially in Indian population.

Methods: The present study is a randomized controlled trial, comparing SR (n=17) and MR ( $\mathrm{n}=13)$ knee kinematics based on clinical scores (Knee Society Score; KSS), radiological (Lotke score), fluoroscopic, and electro-goniometric data in patients. Fluoroscopic evaluation was done by contour based (2D/3D) registration technique.

Results: Patients demographics, KSS, radiological and electro-goniometric data were comparable between SR and MR TKA groups. Anatomically, axial rotation and condylar lift-off data showed insignificant differences. However, MR TKA knees showed higher $(\mathrm{P}<0.05)$ antero-posterior translation compared to SR TKA knees. Similarly, electro-goniometry data has shown no statistical significant difference between both the groups in terms of unloaded flexion-extension, internal-external rotations, walking, and sitto-stand flexion/rotation.
\end{abstract}

Conclusion: The study showed no statistical significant difference between the two knee implant designs based on clinical, radiological and goniometric data except antero-posterior translation. Mid-flexion instability was not seen with MR TKA which was projected as the major problem with these designs.

Keywords: Electrogoniometry; Fluoroscopy; Knee Society Score; TKA; 2D over 3D registration.

\section{Introduction}

Total Knee Arthroplasty (TKA) is an established procedure for end-stage osteoarthritis of knee joint which relieves patients' pain and improves the quality of life [1-3]. A successful TKA demands precise surgical technique, appropriate materials, sound implant design and kinematics, and patient compliance with rehabilitation [4]. Implant design has undergone tremendous changes in the past few years. Initially, multi radii (MR) TKA knee implant was designed based on the hypothesis that normal knee joints have multiple instantaneous centres of flexion-extension [5,6]. However, the concept has been challenged by recent studies which propose a constant centre of rotation, hence the basis of newly introduced single radius (SR) implant designs [7-9]. An important aim of TKA is in replicating the normal knee kinematics $[4,10,11]$, which helps in increasing the longevity of implant and eventually patient satisfaction.

Mid-flexion instability and quadriceps loading were the primary hurdles which were proposed to be averted with new SR implants [11-13]. Other proposed biomechanical advantage with 
the SR design was that the knee flexion-extension axis is situated more posteriorly in the SR design than the MR TKA design that leads to longer quadriceps moment arm and hence making it biomechanically more efficient [11]. The SR TKA has also been reported to improve anterior knee function, stability, and flexion with greater proprioception than MR implants [12,13]. However, limited or no data is available on the head-on comparison of SR and MR implant's kinematics in TKA patients post-surgery especially in Indian population. In the present randomized control trial, we compared SR and MR implant designs using clinical, radiological and goniometric analysis in Indian patients who were undergone TKA. We used 2D fluoroscopy over implant 3D registration technique (Mimics 17.0 software, Materialise NV, Leuvan, Belgium) to visualize implants structures and relative position and electrogoniometry to analysis functional parameters of knees.

\section{Material and Methods}

\section{Patients}

Patients (age 50-70 years) who were undergoing TKA due to the primary osteoarthritis of the knee between October 2013 and December 2014 were included in the trial. Patients were initially randomized patient-wise to either SR or MR groups until each group had at least 12 knees. If a patient was undergoing a bilateral TKA, then both knees were implanted with same type of implant (SR/MR) and counted as two individual knees. The key inclusion criteria were patients with primary osteoarthritis of single or both knee with Kellgren Lawrence classification grade $\geq 3$; at least 3 to 4 months follow-up post TKR; Knee Society Score (KSS) $>70$ and Lotke radiological score $>80,3$ months post TKR; at least 3 months gap post TKA for sequential bilateral TKA. The key exclusion criteria were patients with secondary osteoarthritis; hip arthritis and previously operated hip conditions; any deformity of foot or ankle; lost to follow-up; dementia; neuropathic joints; revision knee Arthroplasty; and history of septic arthritis in the same knee.

\section{Ethics}

The studies were conducted in accordance with Good Clinical Practice, ISO 14155, Institutional Ethics Committee, and in compliance with the approved protocol and applicable regulatory requirements. All patients provided written informed consent before entering the study.

\section{Study Design and Settings}

The present study was a randomized controlled trial, comparing in vivo SR and MR knee kinematics based on clinical score, radiological scores, fluoroscopic, and electro-goniometric data. The study was conducted in tertiary care orthopaedic centres in India.

\section{Study Treatments}

Radiographs of bilateral knee antero-posterior and lateral views were taken for all the patients along with a standing scanogram to calculate the mechanical and anatomical axes. Grading of osteoarthritis was done according to Kellgren Lawrence classification [14]. All the eligible patients were randomized in to two groups, SR TKA and MR TKA. Knees in SR TKA group were implanted with Scorpio Single Axis implants (Stryker, Orthopaedics, USA), whereas knees in MR TKA group implanted with Genesis II implants (Smith \&Nephew, USA).

All patients were operated by the same surgeon using standard medial parapatellar approach under tourniquet cover. On the day 1 of post-surgery, patients were mobilized with brace and knee range of motion exercises (flexion-extension) and quadriceps exercises were initiated. After 3 months of follow-up, post-TKA fresh radiographs and scanogram of knee were taken and patient were evaluated against the clinical (KSS) [15] and radiological score (Lotke) [16]. All the eligible patients with clinical score of $\geq 70$ and radiological score of $\geq 80$ were taken up for further assessments.

\section{Study Assessments}

We used earlier reported contour matching technique, also known as $2 \mathrm{D} / 3 \mathrm{D}$ registration technique, to analyze the position of implants in vivo [17]. A library of computer aided design (CAD) models of silhouette was built on 3D images of prostheses obtained from digitized single-view fluorescence 2D images of prostheses (MIMICS software, Materialise NV, Leuvan, Belgium). The CAD generated silhouette images were superimposed on corresponding fluoroscopic image extracted silhouette to evaluate relative motion of femur and tibia as per the standard joint coordinate systems (Grood and Suntay)[18].

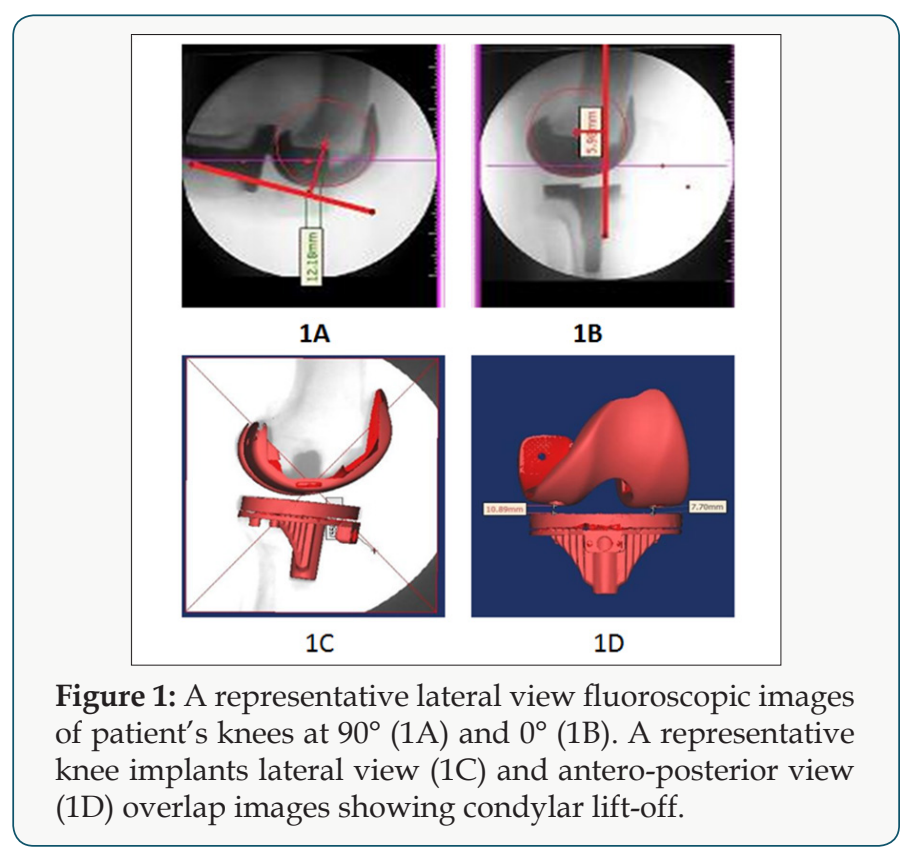

Antero-Posterior Translation: Antero-posterior translation of femoral component was evaluated by comparing the femoral component movement using lateral fluoroscopic views $\left(0^{\circ}\right.$ and $\left.90^{\circ}\right)$ at a $90^{\circ}$ flexion (Figures $1 \mathrm{~A} \& 1 \mathrm{~B}$ ). A circle was drawn to best fit the femoral component and the centre of circle position was analyzed in $0^{\circ}$ and $90^{\circ}$ with respect to the anterior margin of the tibial peg, a reference line. 
Femoral Component Lift-Off: The femoral component lift-off was calculated as the distance between medial and lateral condyles from tibial plateau in the superimposed image. For calculations, the superimposed images were rotated by $90^{\circ}$ to obtain the anteroposterior view (Figures 1C \&1D), especially at $30^{\circ}$ and $60^{\circ}$ flexion to evaluate mid-flexion instability.

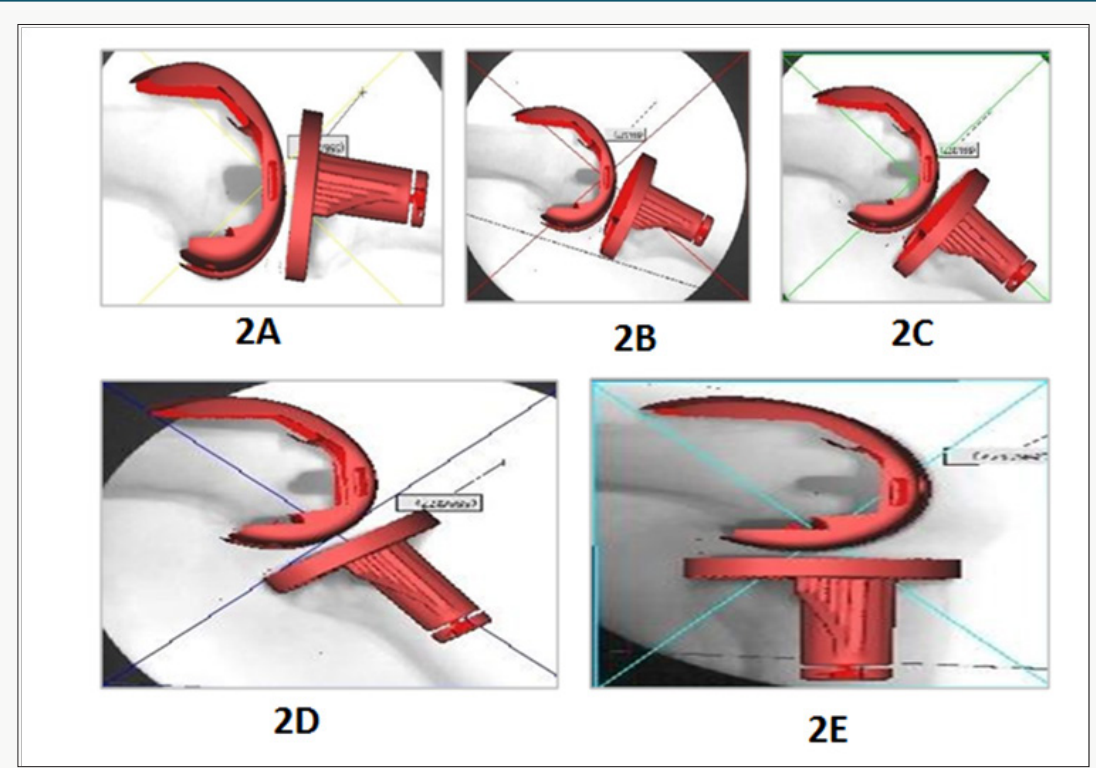

Figure 2: A representative image of femoral and tibial component $3 \mathrm{D}$ position at $0^{\circ}(2 \mathrm{~A}), 30^{\circ}(2 \mathrm{~B}), 45^{\circ}(2 \mathrm{C}), 60^{\circ}(2 \mathrm{D})$, and $90^{\circ}(2 \mathrm{E})$.

Tibio-Femoral Rotation: The rotation of femoral and tibial components along with internal-external rotation axis and varus/ valgus rotation axis was analyzed by calculating Tibio-femoral relation. The superimposed images were analyzed to find out the 3D position of implants in space. The final 3D position of the femoral and tibial component were analyzed with respect to their base value and then finally with respect to each other at $0^{\circ}, 30^{\circ}, 45^{\circ}$, $60^{\circ}$ and $90^{\circ}$ (Figures 2A-2E).

Flexion-Extension Instability: Flexion-extension instability was calculated on fluoroscopic antero-posterior valgus-varus stress view images. To overcome the magnification errors, we calculated the angular joint line opening on these views rather than the linear measurements (Figures 3A \& 3B).

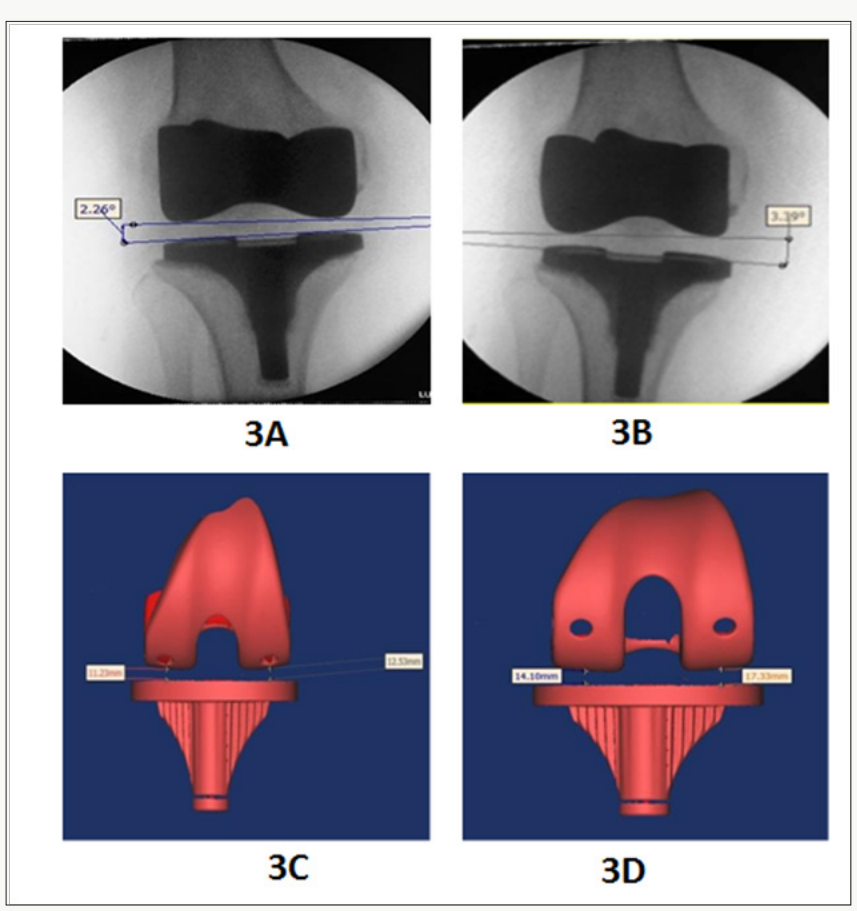

Figure 3: Fluoroscopic images showing valgus stress or medial joint line opening (3A) and varus stress view or lateral joint line opening (3B). Representative images showing condylar lift-off at $30^{\circ}(3 \mathrm{C})$ and $60^{\circ}(3 \mathrm{D})$. 


\section{Statistical analysis}

All the data was validated and checked for its veracity; including the outliers. All measurement data (discrete and continuous) was reported in terms of mean and standard deviation. All the categorical data was reported as frequency. The significance analysis was performed with a paired t-test between the arms.

\section{Results}

\section{Patient Demographics}

Twenty three patients were evaluated for eligibility criteria, and all the patients met the criteria. Randomly, 15 patients were randomized into SR group and 7 patients were randomized into MR group to make sure an equal number of knees were allocated to each group. MR group had lesser patients compared to SR because most of MR group patients were undergoing bilateral TKA hence the double the number of knees. Four patients were excluded from the study; 3 patients did not meet criteria and 1 patient did not meet KSS score (Figure 4). Three months post TKA follow-up, 19 eligible patients ( 8 male, 11 female) were included in the present study. Of these patients, 11 patients had bilateral and 8 patients had single knee TK Aculminating to a total of 30 knee replacements. Seventeen knees were treated with SR implants ( 5 male, 7 female patients) and rest of the 13 knees were treated with MR implants ( 3 male, 4 female patients), and none of the bilateral patients had both kinds (SR and MR) of implants. The study included 14 male (9 SR TKA, 5 MR TKA) and 16 female knees (8 SR TKA, 8 MR TKA).There were 8 left and 9 right knees in SR TKA group, whereas 7 left and 6 right knees in MR TKA group. Both the patients groups were similar in demographics and diagnosis (Table 1).

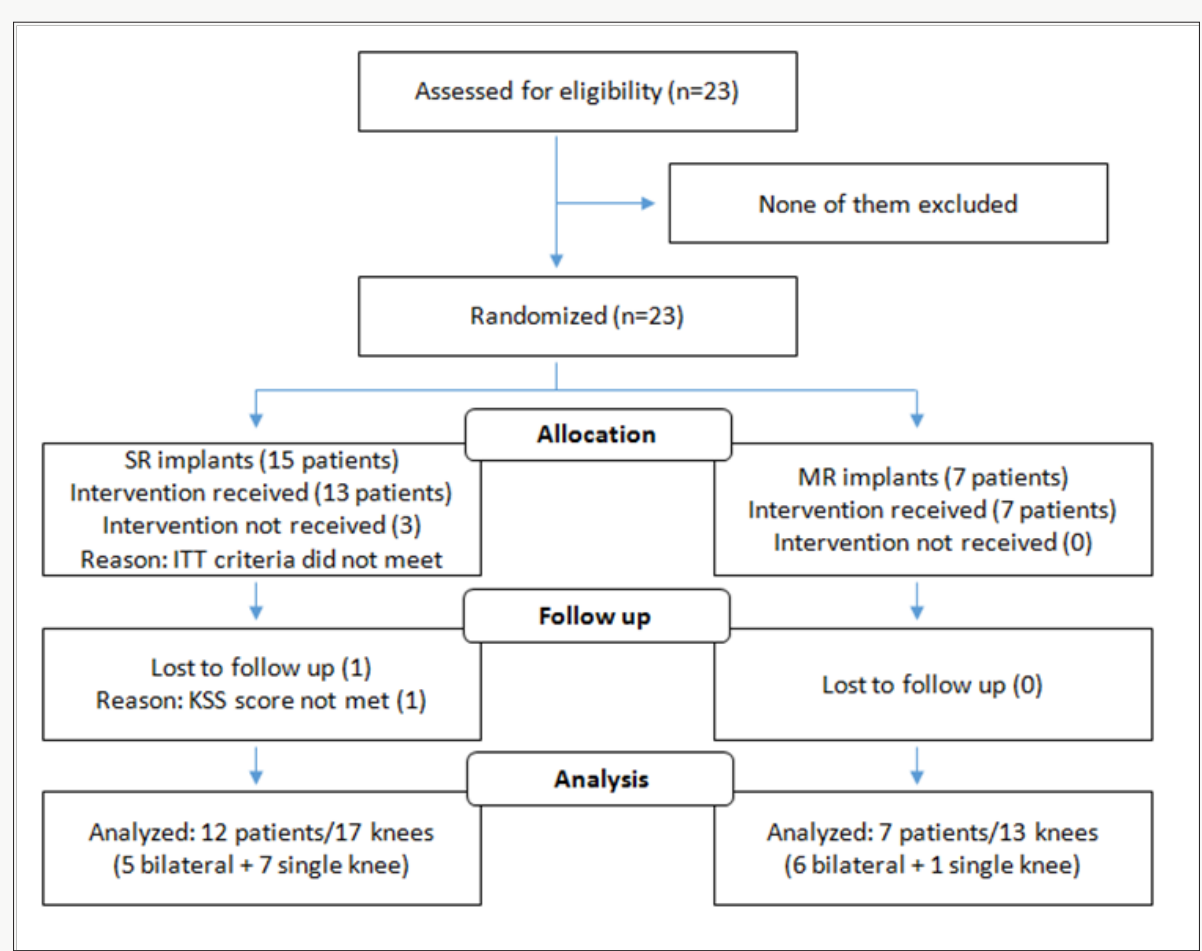

Figure 4: Consort chart of patient's randomization, allocation, and analysis.

Table 1: Patients Demographics.

\begin{tabular}{|c|c|c|c|}
\hline Parameter & SR TKA & MR TKA & P value \\
\hline Male & 5 & 3 & \\
\hline Female & 7 & $62.53 \pm 8.38$ & \\
\hline Age $($ mean \pm SD) & $62.29 \pm 8.48$ & 5 & \\
\hline Male knees & 9 & 8 & 7 \\
\hline Female knees & 8 & 6 & \\
\hline Left TKA & 8 & $66.31 \pm 25.18$ & \\
\hline Right TKA & $95.18 \pm 23.81$ & $152.77 \pm 19.73$ & $>0.05$ \\
\hline Pre-TKA KSS (mean \pm SD) & $146.24 \pm 14.03$ & $83 \pm 1.87$ & $>0.05$ \\
\hline Post-TKA KSS (mean \pm SD) & $83.18 \pm 1.96$ & & \\
\hline Lotke Score $($ mean SD) & & & \\
\hline
\end{tabular}

KSS-Knee Society Score; MR -Multi radii implant; SD -Standard deviation; SR -Single radius implant; TKA-Total Knee Arthroplasty. 
KSS and Lotke Scores: The mean post-operative KSS was improved to $146.24 \pm 14.03$ and $152.77 \pm 19.73$ from mean baseline pre-operative score $95.18 \pm 23.81$ and $66.31 \pm 25.18$ (P > 0.05) for SR and MR TKA, respectively. The post-operative radiological Lotke Score was $83.18 \pm 1.96$ and $83 \pm 1.87(\mathrm{P}>0.05)$ in SR and MR TKA knees, respectively (Table 1 ).

Anatomical Assessments: The mean antero-posterior translation of SR and MR TKA knees was $6.77 \pm 2.34 \mathrm{~mm}$ and $9.00 \pm 2.95 \mathrm{~mm}(\mathrm{P}>0.05)$, respectively, at a knee flexion of $90^{\circ}$. Overall, about 36\% TKA knees (7 SR and 4 MR TKA) showed medial condylar lift-off, whereas about 53\% TKA knees (10 SR and 6 MR TKA) showed lateral condylar lift-off at $30^{\circ}$ (Figure 3C); $10 \%$ TKA knees did not show any lift-off and all are from MR TKA knee group. At $60^{\circ}$, about $36 \%$ TKA knees (6 SR and 5 MR TKA) showed medial condylar lift-off, whereas about 53\% TKA knees (10 SR TKA, 7 MR TKA) showed lateral condylar lift-off (Figure 3D); 2 TKA knees did not show lateral condylar lift-off, one from each group (Table 2).The average lift-off values in SR and MR TKA knees were $3.20 \pm$ $2.19 \mathrm{~mm}$ and $2.16 \pm 2.41 \mathrm{~mm}$ at $30^{\circ}$ and $3.10 \pm 1.69 \mathrm{~mm}$ and $3.68 \pm$ $2.63 \mathrm{~mm}$ at $60^{\circ}$, respectively. In Valgus-varus stress test, SR and MR TKA knees showed medial joint line opening of $1.84^{\circ} \pm 1.14^{\circ}$ and $2.42^{\circ} \pm 1.38^{\circ}$, and lateral joint opening of $2.12^{\circ} \pm 1.42^{\circ}$ and $1.90^{\circ}$ $\pm 1.31^{\circ}$, respectively. The Axial rotation of all the patients were evaluated after simulation/superimposition at $30^{\circ}, 45^{\circ}, 60^{\circ}$ and $90^{\circ}$ and compared with the position at $0^{\circ}$ (Figure 5A-5E). The average axial rotation was $3.63^{\circ}$ in SR TKA group and $1.51^{\circ}$ in MR TKA group. Ten TKA knees each from SR and MR TKA knees followed normal internal rotation of tibia at $0-90^{\circ}$, whereas 7 of 17 SR TKA knees and 3 of 13 MR TKA knees had external rotation of tibia in flexion (reverse rotation). The range of rotation varied from $23.31^{\circ}$ to $-27.40^{\circ}$ and $15.76^{\circ}$ to $-15.5^{\circ}$ for SR TKA and MR TKA knees, respectively, across all degrees of flexion (Table 2).

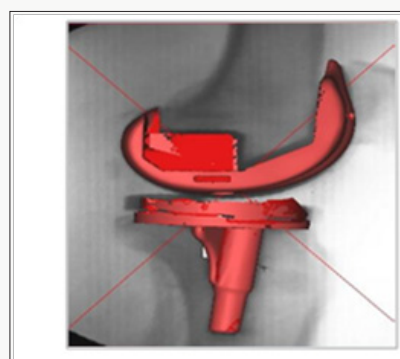

5A

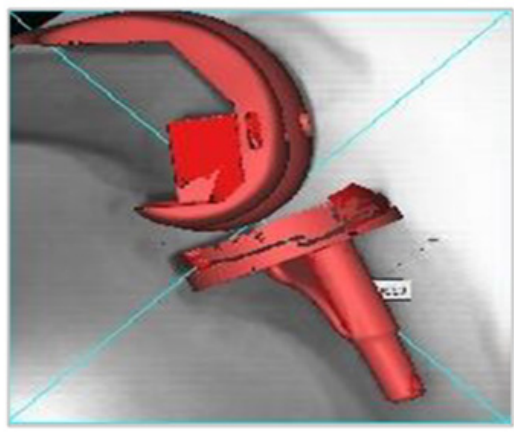

5D

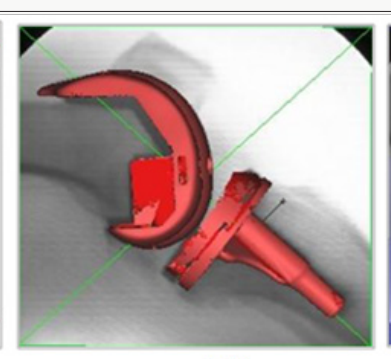

5B

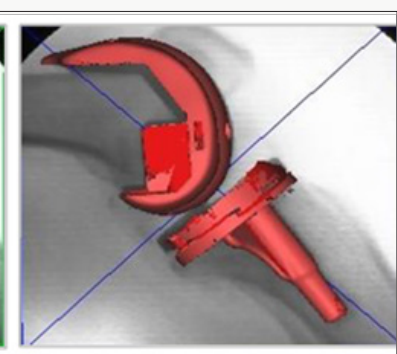

$5 C$

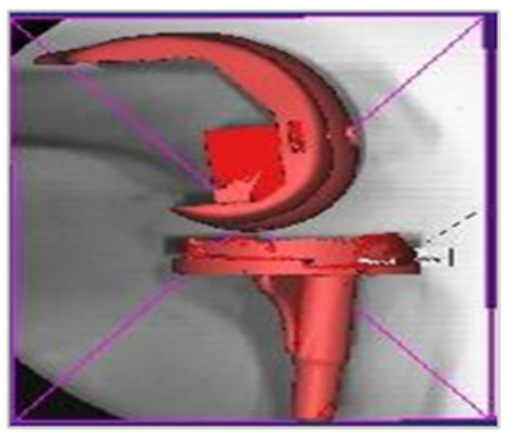

$5 \mathrm{E}$

Figure 5: A representative images of axial rotation at $0^{\circ}(5 \mathrm{~A}), 30^{\circ}(5 \mathrm{~B}), 45^{\circ}(5 \mathrm{C}), 60^{\circ}(5 \mathrm{D})$, and $90^{\circ}(5 \mathrm{E})$.

Table 2: Fluoroscopic Assessments.

\begin{tabular}{|c|c|c|c|}
\hline Parameter & SR TKA & MR TKA & P value \\
\hline AP translation(mean $\pm \mathrm{SD})$ & $6.77 \pm 2.34^{\circ}$ & $9.00 \pm 2.95^{\circ}$ & $<0.05$ \\
\hline Lift-off at $30^{\circ}$ Medial (\#knees) & 7 & 4 & \\
\hline Lift-off at $30^{\circ}$ Lateral (\#knees) & 10 & 6 & \\
\hline No lift-off at $30^{\circ}$ (\#knees) & 0 & 3 & \\
\hline Lift-off at $60^{\circ}$ Medial (\#knees) & 6 & 5 & \\
\hline Lift-off at $60^{\circ}$ Lateral (\#knees) & 10 & 7 & \\
\hline No lateral condylar lift-off at $60^{\circ}$ (\#knees) & 1 & 1 & \\
\hline Average lift-off at $30^{\circ}, \mathrm{mm}$ & $3.20 \pm 2.19$ & $2.16 \pm 2.41$ & $>0.05$ \\
\hline Average lift-off at $60^{\circ}, \mathrm{mm}$ & $3.10 \pm 1.69$ & $3.68 \pm 2.63$ & $>0.05$ \\
\hline
\end{tabular}

Citation: Lalit M, Ankur S, Anshul G, Amit S, Surabhi R, et al. Evaluation of Knee Kinematics in Single Radius versus Multi-Radii Total Knee Arthroplasty in India Population: A Randomized Control Trial. Op Acc J Bio Eng \& Bio Sci 1(3)- 2018. OAJBEB.MS.ID.000113. DOI: 10.32474/OAJBEB.2018.01.000112. 


\begin{tabular}{|c|c|c|c|}
\hline Valgus Instability (mean \pm SD) & $1.84 \pm 1.14^{\circ}$ & $2.42 \pm 1.38^{\circ}$ & $>0.05$ \\
\hline Varus instability $($ mean \pm SD $)$ & $2.12 \pm 1.42^{\circ}$ & $1.9 \pm 1.31^{\circ}$ & $>0.05$ \\
\hline Average rotation & $3.63^{\circ}$ & $1.51^{\circ}$ & \\
\hline Normal rotation of tibia $\left(0-90^{\circ}\right)$ (\#knees) & 10 & 10 & \\
\hline External rotation of tibia $\left(0-90^{\circ}\right)$ (\#knees) & 7 & 3 & \\
\hline $\begin{array}{l}\text { *Average max normal rotation, any } \\
\text { increment }\end{array}$ & $10.98^{\circ}$ & $8.44^{\circ}$ & \\
\hline $\begin{array}{l}{ }^{* *} \text { Average max reverse rotation, any } \\
\text { increment }\end{array}$ & $-7.21^{\circ}$ & $-4.98^{\circ}$ & \\
\hline Maximum normal rotation, any increment & $23.31^{\circ}$ & $15.76^{\circ}$ & \\
\hline Maximum reverse rotation, any increment & $-27.4^{\circ}$ & $-15.5^{\circ}$ & \\
\hline
\end{tabular}

MR -Multi radii implant; SD -Standard deviation; SR -Single radius implant; TKA -Total Knee Arthroplasty

* Internal rotation of tibia with flexion (normal rotation)

**External rotation of tibia with flexion (reverse rotation).

Functional Assessments: In unloaded flexion-extension, SR TKA knees had an active and passive range of motion (ROM) of $107 \pm 20.36^{\circ}$ and $115.27 \pm 21.34$ compared to $120.47 \pm 17.93^{\circ}$ and $126.93 \pm 23.14^{\circ}$ for MR TKA knees, respectively. In unloaded internal-external rotations, the mean axial rotations in SR TKA knees while performing active and passive ROM was $29.78 \pm 16.19^{\circ}$ and $32.82 \pm 10.92^{\circ}$ compared to $21.32 \pm 6.67^{\circ}$ and $26.65 \pm 7.94^{\circ}$ Table 3: Electrogionometry Assessments.

\begin{tabular}{|c|c|c|c|}
\hline Parameter & SR TKA $\quad$ (mean \pm SD) & MR TKA (mean \pm SD) & P value \\
\hline Unloaded Flexion-Extension & & & $>0.05$ \\
\hline Active ROM & $107.54 \pm 20.36^{\circ}$ & $120.47 \pm 17.93^{\circ}$ & $>0.05$ \\
\hline Passive ROM & $115.27 \pm 21.34^{\circ}$ & & \\
\hline $\begin{array}{c}\text { Unloaded Internal-External } \\
\text { Rotations }\end{array}$ & & $21.31 \pm 6.67^{\circ}$ & $>0.05$ \\
\hline Rotations unloaded active ROM & $29.78 \pm 16.19^{\circ}$ & $26.65 \pm 7.94^{\circ}$ & $>0.05$ \\
\hline Rotations unloaded passive ROM & $32.82 \pm 10.92^{\circ}$ & & $>0.05$ \\
\hline Walking & & $63.83 \pm 15.2^{\circ}$ & $>0.05$ \\
\hline Loaded walking (flexion) & $60.35 \pm 9.46^{\circ}$ & $30.05 \pm 12.32^{\circ}$ & \\
\hline Loaded walking (rotation) & $31.05 \pm 8.13^{\circ}$ & & $>0.05$ \\
\hline Sit-to-stand (STS) & & $84.18 \pm 13.35^{\circ}$ & $>0.05$ \\
\hline Loaded STS (flexion) & $77.8 \pm 9.44^{\circ}$ & $34.96 \pm 6.31^{\circ}$ & \\
\hline Loaded STS (rotation) & $28.03 \pm 13.74^{\circ}$ & & \\
\hline
\end{tabular}

MR -Multi radii implant; SD-Standard deviation; SR-Single radius implant; TKA-Total Knee Arthroplasty.

\section{Discussion}

The present was a randomized control trial compared the anatomical and functional parameters of the single and multi-radii TKA implants in Indian patients. Based on clinical, radiological, and goniometric data our study showed no significant difference between the SR and MR knee implant designs in terms of anatomical and functional parameters, except antero-posterior translation. To ensure similar knees were included in both the groups, patients who met KSS and Lotke score, 3 months post-TKA, were included in the study assessments. Patient's KSS score was improved in the post- for MR TKA knees, respectively (Table 3). While walking on a flat surface, SR TKA knees had $60.35 \pm 9.46^{\circ}$ flexion with a mean axial rotation of $31.05 \pm 8.13^{\circ}$, whereas MR TKA knees had $63.83 \pm 15.20^{\circ}$ flexion and axial rotation of $30.05 \pm 12.32^{\circ}$. During sitting to standing movements, SR TKA knees had a flexion of $77.8 \pm 9.44^{\circ}$ with an axial rotation of $28.03 \pm 13.74^{\circ}$, whereas MR TKA knees had a flexion of $84.18 \pm 13.35^{\circ}$ with an axial rotation of $34.96 \pm 6.31^{\circ}$ (Table 3 ). 
significantly higher $(\mathrm{P}>0.05)$ antero-posterior translation from $0-90^{\circ}$ knee flexion than the SR implants and both groups anteroposterior translation was within the range of earlier reported studies [19-21]. Higher antero-posterior translation in MR knees indicates better replication of normal knee kinematics and more posterior roll back was possible with no instability compared to SR knees. The present study's valgus and varus instability (medial/ lateral joint line opening in extension) for SR and MR TKA knees were closer to earlier reported values of $4^{\circ}$ and $2^{\circ}$ [22], respectively. However, there was no statistically significant difference between both the groups in either valgus or varus stress $(\mathrm{P}<0.05)$ test indicating that both the groups were matched in their soft tissue balancing.

Although implant design has limited role in femoral component lift-offand primarily depends on softtissue balancing and component placement, knees from both the groups were showing either medial or lateral condylar lift-off at both $30^{\circ}$ and $60^{\circ}$. The average condylar lift-off for both SR and MR implant was within the earlier reported average range of $<2 \mathrm{~mm}$ to $8 \mathrm{~mm}$ [23]. There was no statistically significant difference between the groups in condylar lift-off. We used electro-goniometry, a previously validated technique, to compare SR and MR TKA knees functionality [24]. Although, MR TKA knees average active and passive ROM was relatively higher than the SR TKA knees, but there was no statistically significant difference between SR and MR TKA knees in term of unloaded flexion-extension active $(\mathrm{P}>0.05)$ or passive $(\mathrm{P}>0.05)$ ROM. However, both SR and MR TKA knees ROM was similar to earlier reported studies [25]. Similarly, there was no statistical difference in the axial Tibio-femoral rotation during either active or passive ROM ( $P>0.05$ and $P>0.05$, respectively) between the SR and MR TKA knee groups. Knee excursion while walking and sit-to-stand were well within the earlier reported values [25-27], however there was no statistically significant difference between SR and MR TKA knee excursion.

Overall, our study corroborated earlier studies [28,29] and showed no statistical difference between SR and MR implant in terms of anatomical and functional parameters, however MR implants had better antero-posterior translation than the SR implants. In agreement with earlier reported studies [30], our analysis did not find any mid-range instability of the knees and could not demonstrate enhanced mid-range stability of the SR TKA over the MR TKA.

\section{Conclusion}

The present randomized control trial did not show any statistically significant difference between SR and MR implants in terms of knee kinematics based on the fluoroscopic, radiological and goniometrical data except antero-posterior translation. However, conclusion should be interpreted carefully as study has a limitation of fewer subjects.

\section{References}

1. Robertsson O, Dunbar M, Pehrsson T, Knutson K, Lidgren L (2000) Patient satisfaction after knee arthroplasty: a report on 27,372 knees operated on between 1981 and 1995 in Sweden. Acta orthopaedica Scandinavica 71(3): 262-267.

2. Mahomed N, Gandhi R, Daltroy L, Katz JN (2011) The self-administered patient satisfaction scale for primary hip and knee arthroplasty. Arthritis 2011: 1-6.

3. Bourne RB, Chesworth BM, Davis AM, Mahomed NN, Charron KD (2010) Patient satisfaction after total knee arthroplasty: who is satisfied and who is not? Clinical orthopaedics and related research 468(1): 57-63.

4. Riley LH (1976) The evolution of total knee arthroplasty. Clinical orthopaedics and related research 120: 7-10.

5. Gunston FH (1971) Polycentric knee arthroplasty. Prosthetic simulation of normal knee movement. The Journal of bone and joint surgery British 53(2): 272-277.

6. Frankel VH, Burstein AH, Brooks DB (1971) Biomechanics of internal derangement of the knee. Pathomechanics as determined by analysis of the instant centers of motion. The Journal of bone and joint surgery American 53(5): 945-962.

7. Hollister AM, Jatana S, Singh AK, Sullivan WW, Lupichuk AG (1993) The axes of rotation of the knee. Clinical orthopaedics and related research 290: $259-268$

8. Churchill DL, Incavo SJ, Johnson CC, Beynnon BD (1998) The transepicondylar axis approximates the optimal flexion axis of the knee. Clinical orthopaedics and related research 356: 111-118.

9. Iwaki H, Pinskerova V, Freeman MA (2000) Tibiofemoral movement 1: the shapes and relative movements of the femur and tibia in the unloaded cadaver knee. The Journal of bone and joint surgery British 82(8): 1189-1195.

10. Hawker G, Wright J, Coyte P, Paul J, Dittus R, et al. (1998) Health-related quality of life after knee replacement. The Journal of bone and joint surgery American 80(2): 163-173.

11. Lima DD, PooleC, Chadha H, Hermida JC, Mahar A, etal. (2001) Quadriceps moment arm and quadriceps forces after total knee arthroplasty. Clinical orthopaedics and related research 392: 213-220.

12. Ezechieli M, Dietzek J, Becher C, Ettinger M, Calliess T, et al. (2012) The influence of a single-radius-design on the knee stability. Technology and health care: official journal of the European Society for Engineering and Medicine 20(6): 527-534.

13. Cook LE, Klika AK, Szubski CR, Rosneck J, Molloy R, et al. (2012) Functional outcomes used to compare single radius and multiradius of curvature designs in total knee arthroplasty. The journal of knee surgery 25(3): 249-253.

14. Kellgren JH, Lawrence JS (1957) Radiological assessment of osteoarthrosis. Annals of the rheumatic diseases 16(4): 494-502.

15. Insall JN, Dorr LD, Scott RD, Scott WN (1989) Rationale of the Knee Society clinical rating system. Clinical orthopaedics and related research 248: 13-14.

16. Ecker ML, Lotke PA, Windsor RE, Cella JP (1987) Long-term results after total condylar knee arthroplasty. Significance of radiolucent lines. Clinical orthopaedics and related research 216: 151-158.

17. Banks SA, Hodge WA (1996) Accurate measurement of three-dimensional knee replacement kinematics using single-plane fluoroscopy. IEEE transactions on bio-medical engineering 43(6): 638-649. 
18. Grood ES, Suntay WJ (1983) A joint coordinate system for the clinical description of three-dimensional motions: application to the knee. Journal of biomechanical engineering 105(2): 136-144.

19. Tanifuji O, Sato T, Kobayashi K, Mochizuki T, Koga Y, et al. (2011) Threedimensional in vivo motion analysis of normal knees using single-plane fluoroscopy. Journal of orthopaedic science : official journal of the Japanese Orthopaedic Association 16(6): 710-718.

20. Asano T, Akagi M, Tanaka K, Tamura J, Nakamura $T$ (2001) In vivo three-dimensional knee kinematics using a biplanar image-matching technique. Clinical orthopaedics and related research 388: 157-166.

21. Pinskerova V, Iwaki H, Freeman MA (2000) The shapes and relative movements of the femur and tibia at the knee. Der Orthopade 29(suppl 1) S3: S5.

22. Romero J, Stahelin T, Binkert C, Pfirrmann C, Hodler J, et al. (2007) The clinical consequences of flexion gap asymmetry in total knee arthroplasty. The Journal of Arthroplasty 22(2): 235-240.

23. Dennis D, Komistek R, Scuderi G, Argenson JN, Insall J, et al. (2001) In vivo three-dimensional determination of kinematics for subjects with a normal knee or a unicompartmental or total knee replacement. The Journal of bone and joint surgery American 83A(2): 104-115.

24. Walker CR, Myles C, Nutton R, Rowe P (2001) Movement of the knee in osteoarthritis. The use of Electrogoniometry to assess function. The Journal of bone and joint surgery British 83(2): 195-198.
25. Myles CM, Rowe PJ, Walker CR, Nutton RW (2002) Knee joint functional range of movement prior to and following total knee arthroplasty measured using flexible Electrogoniometry. Gait \& posture 16(1): 46-54.

26. Rowe PJ MC, Hillmann SJ, Hazlewood ME (2001) Validation of flexible electro goniometry as a measure of joint kinematics. Physiotherapy 87(9): 479-488.

27. van der Linden ML, Rowe PJ, Nutton RW (2008) Between-day repeatability of knee kinematics during functional tasks recorded using flexible Electrogoniometry. Gait \& posture 28(2): 292-296.

28. Jo AR, Song EK, Lee KB, Seo HY, Kim SK, et al. (2014) A comparison of stability and clinical outcomes in single-radius versus multi-radius femoral design for total knee Arthroplasty. The Journal of Arthroplasty 29(12): 2402-2406.

29. Liu S, Long H, Zhang Y, Ma B, Li Z (2016) Meta-Analysis of Outcomes of a Single-Radius Versus Multi-Radius Femoral Design in Total Knee Arthroplasty. The Journal of Arthroplasty 31(3): 646-654.

30. Stoddard JE, Deehan DJ, Bull AM, McCaskie AW, Amis AA (2013) The kinematics and stability of single-radius versus multi-radius femoral components related to mid-range instability after TKA. Journal of orthopaedic research : official publication of the Orthopaedic Research Society 31(1): 53-58.

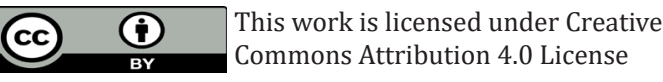

To Submit Your Article Click Here: Submit Article

DOI: $10.32474 /$ OAJBEB.2018.01.000112

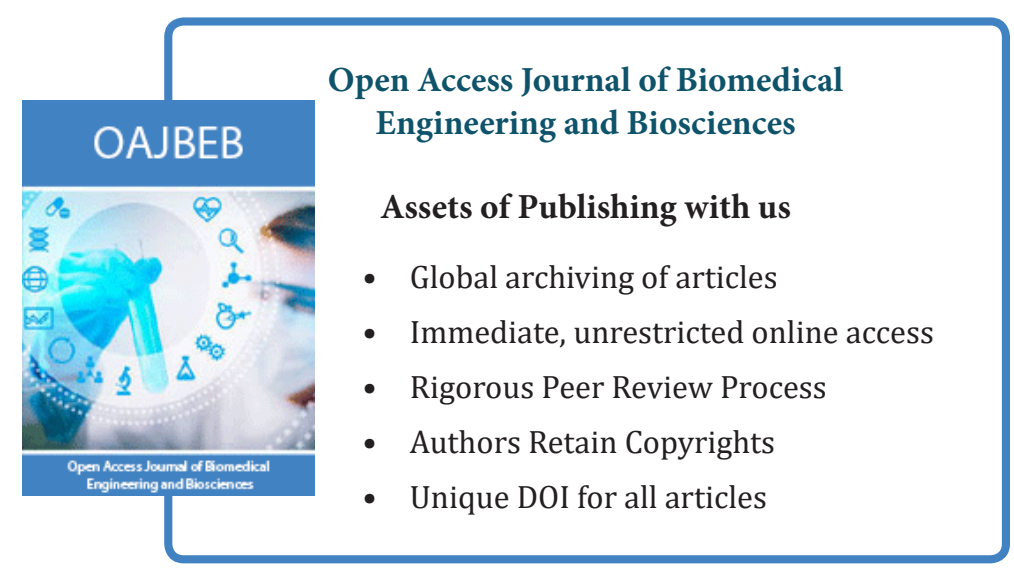

